\title{
Teoría social y régimen de conocimiento. Una crítica al poscolonialismo latinoamericano
}

\author{
Cristóbal Villalobos Dintrans ${ }^{1}$
}

\author{
Fecha de recepción: 22 de mayo de 2017
}

Fecha de aprobación: 31 de julio de 2017

\begin{abstract}
Resumen
El presente artículo analiza la relación contemporánea entre las características societales de América Latina y la producción de teoría social que se genera en el continente. A partir de una breve descripción de una de las respuestas teóricas más desarrolladas y difundidas en el continente durante las últimas décadas, el poscolonialismo, se entiende el problema de la producción de conocimiento como una interrelación entre sociedad y teoría social. El análisis del caso latinoamericano muestra, por un lado, un vacío teórico en el continente, $\mathrm{y}$, por otro, la existencia de un régimen de conocimiento precario, débil y poco desarrollado. Esto nos lleva a repensar el problema de la teoría social latinoamericana desde una nueva perspectiva, que reconozca la existencia de la ciencia universal, abandonando la idea misma de teoría social latinoamericana y reemplazándola por la idea de teoría social realizada por latinoamericanos.
\end{abstract}

Palabras clave: teoría social, poscolonialismo, régimen de conocimiento, América Latina, producción de conocimiento.

\section{Social theory and knowledge regime. Reviewing the Latin American post-colonialism}

\begin{abstract}
This article analyzes the contemporary relationship between the social characteristics of Latin America and the production of social theory generated in the Continent. By a short description of one of the most developed and widespread theoretical responses on the Continent during the last decades -postcolonialism- we understand the issue of knowledge production as interrelated between society and social theory. On the one hand, the analysis of the Latin American case shows a theoretical void in the Continent, and, on the other, the existence of a knowledge regime that is precarious, weak and underdeveloped. This leads us to think again about the subject of Latin Ameri-
\end{abstract}

1 Dr (c) en Ciencias Sociales, Universidad de Chile. Investigador asociado del Centro de Estudios de Políticas y Prácticas en Educación (CEPPE - UC), Pontificia Universidad Católica de Chile. Contacto: clvillal@uc.cl. 
can social theory from a new perspective that recognizes the existence of a universal science abandoning the very idea of Latin American social theory and replacing it with the idea of social theory carried out by Latin Americans.

Keywords: Social theory, post-colonialism, knowledge regime, Latin America, knowledge production.

\title{
Teoria social e regimem do conhecimento. Uma crítica ao poscolonialismo latino-americano
}

\begin{abstract}
Resumo
O presente artigo analisa a relação contemporânea entre as características sociais da América Latina e a produção da teoria social gerada no continente. A partir de uma breve descrição de uma das respostas teóricas mais desenvolvidas e difundidas no continente durante as últimas décadas, o pós-colonialismo, entende-se o problema da produção do conhecimento como uma inter-relação entre a sociedade e a teoria social. A análise do caso latino-americano mostra, por um lado, um vácuo teórico no continente e, por outro, a existência de um regime de conhecimento precário, fraco e subdesenvolvido. Isso nos leva a repensar o problema da teoria social latino-americana a partir de uma nova perspectiva, que reconheça a existência da ciência universal, abandonando a própria ideia da teoria social latino-americana e substituindo-a pela ideia da teoria social realizada pelos latino-americanos.
\end{abstract}

Palavras-chave: teoria social, pós-colonialismo, regime do conhecimento, América Latina, produção de conhecimento.

\section{Introducción. Las ciencias sociales y la teoría social en América Latina}

Una característica de las ciencias sociales latinoamericanas, desde su emergencia e institucionalización en la década de los cincuenta del siglo pasado hasta los años noventa, fue la priorización que los investigadores dieron al análisis concreto de las sociedades por sobre los estudios teóricos de lo social. Esto implicó que, tal como indican De Sierra, Garretón, Murmis y Trindade (2007, p. 47), "el eje central [de las ciencias sociales latinoamericanas] fuera teorizar e investigar el tema del cambio social, siendo la sociedad histórica, más que lo social abstracto, el foco central de análisis". Este abandono de la teoría social — que podríamos llamar fundacional— potenció la generación de análisis sobre nuestras sociedades que utilizaron como referencia $-\mathrm{o}$, en 
lenguaje kuhniano, como paradigma- constructos teóricos desarrollados en otros territorios, especialmente en Europa o Estados Unidos. De esta forma, las ciencias sociales latinoamericanas durante parte importante del siglo pasado se construyeron y desarrollaron fundamentalmente a partir de las continuidades y rupturas ${ }^{2}$ establecidas con las corrientes primero positivistas y luego funcionalistas y marxistas (Trindade, 2007; Garretón, 2014; Brunner y Barrios, 1988) ${ }^{3}$.

Esta lógica de operación se vio fuertemente cercenada con la ola dictatorial que se desató en los años setenta y que, casi sin excepciones, disminuyó el potencial teorizador de las ciencias sociales del continente. Posteriormente, con el retorno a la democracia en los países del Cono Sur y la profundización de la globalización económica y cultural durante los años noventa, las ciencias sociales latinoamericanas comienzan un nuevo momento histórico, que puede ser caracterizado por el alejamiento de las grandes teorías (Garretón, Cavarozzi, Cleaves, Gereffi y Hartlyn, 2004), la profundización de los análisis centrados en la democracia (Trindade, 2007) y la consolidación de un sistema de masificación y privatización de la enseñanza superior y de la

2 Evidentemente, esto no implica negar la existencia de corrientes o teorizaciones sociales en el continente durante estas décadas, sino que iluminar la idea de que un rasgo central de estas teorías es su continua referenciación (positiva o negativa) a matrices teóricas importadas. Como muestra Cortés (2012), aportes teóricos, como los de Gino Germani, Enzo Faletto o Roger Vekemans, se realizaron con importantes cuotas de originalidad respecto de las teorías desarrolladas especialmente en Europa, pero en general manteniendo el sustrato epistemológico y ontológico de dichos paradigmas.

3 Quizás la mayor excepción a esta lógica fueron los análisis e ideas de la teoría de dependencia. Si bien es posible reconocer algunas referencias al funcionalismo norteamericano, las teorías de la modernización, el estructuralismo y el marxismo, la teoría de la dependencia latinoamericana puede reconocerse como un constructo teórico/empírico novedoso desarrollado desde el continente. De todas maneras, esto es un tema de debate profundo hasta el día de hoy. Así, por ejemplo, Theotonio dos Santos (1998) realiza un análisis histórico de la teoría de la dependencia, dando cuenta de esta como una construcción teórica diferenciada e innovadora de otras teorías sociales de la época. En contraste, Packenhaum (1992) adopta una postura en la que la teoría de la dependencia aparece como un conjunto holístico de perspectivas epistemológicas, sin que exista un afán por construir una teoría social propiamente tal. 
enseñanza de las humanidades y ciencias sociales (Bernasconi, 2008; González, 2014).

En términos sociopolíticos, este nuevo periodo puede caracterizarse por el intento de instalar un modelo de desarrollo (Portes, 1998) o una matriz sociopolítica (Garretón et al., 2004) de carácter neoliberal. Más allá de los efectos económicos de este proyecto, que tiene más sombras que luces (CEPAL, 2010; Ffrench-Davis, 2014; Riesco, 2013), es claro que los supuestos detrás de la constitución del modelo neoliberal (el interés individual, la búsqueda de ganancia y la privatización de la vida social) no se han enraizado fuertemente en la cultura, vida cotidiana y relaciones de las sociedades latinoamericanas (Portes, 1998; Garretón et al., 2004). Para algunos autores, esta incapacidad de despliegue del modelo neoliberal ha generado un doble vacío de las sociedades latinoamericanas: social y teórico; social, ya que lo que caracterizaría a las sociedades contemporáneas del continente sería que estas "parecieran no tener una problemática central a partir de la cual construir un modelo, teoría o contraproyecto" (De Sierra et al., 2007, p. 52); teórico, ya que la teoría latinoamericana se encontraría sin un referente interpretativo común, privilegiando el desarrollo de múltiples interpretaciones y focos analíticos (Moreno, 1997) y el potenciamiento de las teorías de rango medio (Garretón et al., 2004; Portes, 2004) por sobre la reconstrucción de un paradigma o modelo de análisis de la realidad.

Frente a este escenario, parece relevante preguntarse por la relación contemporánea que podría existir entre las características societales de América Latina y la producción de teoría social que se genera en el continente, lo que constituye el objetivo central de este artículo. Para ello, el texto se estructura en cuatro apartados, adicionales a esta introducción. El segundo apartado describe brevemente algunos de los principales elementos del poscolonialismo que es, desde nuestra perspectiva, una de las respuestas teóricas más desarrolladas y difundidas durante las últimas décadas para responder al anteriormente descrito vacío teórico y societal del continente. A continuación, el tercer aparta- 
do propone un modelo de abordaje para el estudio de la relación entre teoría social y sociedad, a partir de la introducción de la noción de régimen de conocimiento. La incorporación de este concepto permite, en el cuarto apartado, realizar un análisis de los principales elementos que definirían el régimen de conocimiento de las sociedades latinoamericanas, mediante una descripción de tres componentes centrales de estos regímenes: las universidades, los centros de investigación y el rol de la ciencia y tecnología. Considerando lo anterior, el último capítulo desliza algunas reflexiones finales y genera algunas interrogantes respecto de las limitaciones de la mirada poscolonial y la relación entre la teoría social y la estructuración de las sociedades latinoamericanas, entregando una propuesta que permita consolidar y profundizar la producción de conocimiento sobre teoría social en el continente.

\section{Poscolonialidad latinoamericana. ¿Una alternativa al vacío teórico?}

Una de las interpretaciones de la realidad de América Latina que ha surgido con más fuerza durante las últimas décadas (y que sus antecedentes se remontan a mediados del siglo pasado, a través de, por ejemplo, los textos de Franz Fanon, Albert Memmi o Aime Césaire) es la que podríamos agrupar y catalogar bajo el rótulo de "poscolonialidad latinoamericana" ${ }^{4}$. Si bien es claro que existen continuidades con los estudios de la poscolonialidad desarrollados en Asia, África, Europa y Estados Unidos (Bortoluci y Jansen, 2013; Lander, 2000) ${ }^{5}$, lo central de los estudios que

4 Como se indicó, el objetivo del artículo es discutir la relación entre teoría social y sociedad en América Latina, utilizando para ello como marco de referencia las teorías postcoloniales. Por lo mismo, el texto no pretende cubrir ni describir todas las aristas del universo teórico que se podría catalogar como "poscolonialismo", sino dar cuenta de algunos de sus rasgos más genéricos, lo que, casi inevitablemente, implica realizar generalizaciones.

5 Por una parte, Bortoluci y Jansen (2013, pp. 204-216) reconocen cuatro elementos similares entre los investigadores que estudian la poscolonialidad en América Latina respecto de los estudios realizados en otros espacios: i) el cuestionamiento 
se desarrollan desde este programa o paradigma analítico es que buscan explícitamente construir un modelo epistémico, teórico y metodológico particular y nuevo para interpretar la realidad latinoamericana contemporánea (Alarcón, 2001; Lander, 2000; López-Segrera, 2000; Briceño-León y Sonntag, 1998). De esta forma, y tal como dice Alarcón (2001, p. 86), se trata de llenar el vacío analítico y social a partir de una lectura "de Latinoamérica desde ella misma, [que permita] reoriginarizarla, pero no desde otros marcos referenciales" sino a partir de una reflexión propia del continente.

A pesar de este marco común, las fórmulas para realizar esta reconstrucción son múltiples y diversas. Así, por ejemplo, mientras para Briceño-León y Sonntag (1998) lo central del esfuerzo teórico es la generación de una episteme o forma de conocimiento basada en el pueblo, para Boaventura de Sousa Santos (2010) el foco prioritario debe estar puesto en la reconstrucción del conocimiento mediante una ecología de saberes, que permita generar una epistemología del sur (2009) totalmente distinta a las epistemologías occidentales. En una línea complementaria, Lanz (1998) destaca la necesidad de generar una autonomía epistemológica en el continente, que permita fundar espacios analíticos caracterizados por la inter, multi y transdisciplinariedad, mientras que para Zemelman (2005, p. 23) lo central es "pensar que el futuro del continente no puede esbozarse si no es en estrecha relación con las fuerzas sociales y, por lo mismo, no se puede disociar la construcción del pensamiento de los sujetos que construyen historia". A pesar de sus diferencias, estas apuestas analíticas contienen un sustrato común: buscan construir una teoría social latinoame-

a la modernidad occidental; ii) la crítica al provincialismo europeo; iii) el paso de macro-relaciones sociales a una perspectiva que enfatiza las dinámicas sociales, y iv) la necesidad de escribir la historia desde los márgenes. En un sentido distinto, Lander (2000, p. 13) reconoce en las aproximaciones feministas, el cuestionamiento de la historia universal, el orientalismo, los estudios subalternos y los estudios poscoloniales, ciertas semejanzas y similitudes con la producción poscolonial latinoamericana. 
ricana desde, para y con los latinoamericanos. En general, y a riesgo de realizar generalizaciones, creemos que es posible reconocer tres elementos que son relativamente transversales a estas posturas, que definen lo que entenderemos por "poscolonialismo latinoamericano".

En primer lugar, se reconoce que la teoría social poscolonial debe desmarcarse de las lógicas europeizantes de dominación intelectual e interpretación de la realidad. Tal como dice Quijano (2012), esto implica hacer un cuestionamiento del poder/saber/ ser desde el cual se estructura la dominación colonial de la que serían parte las sociedades latinoamericanas — que Mignolo (2000) llama el sistema mundo moderno/colonial-, a partir de la regeneración de una forma propia de conocer y vivir en el mundo. Esto lleva a realizar una crítica radical a los análisis universales y a las concepciones modernas de la realidad (Dussel, 2000), las que son reemplazadas por una mirada que privilegia el relativismo como método de acercamiento ontológico y el localismo como estrategia teórico-metodológica de análisis (Zemelman, 2005; Briceño-León y Sonntag, 1999). Se trata, de esta manera, de una propuesta que critica, en términos epistemológico-conceptuales, las concepciones tradicionales de interpretación de la modernidad, entendiéndolas como un elemento estructurador de la colonialidad (Costa, 2006) ${ }^{6}$. Asimismo, esta crítica a la modernidad implica un fuerte cuestionamiento a la idea típicamente moderna de ciencia. Frente a este escenario, la propuesta poscolonial es la creación de una ciencia nueva, no lineal, mecánica ni minimalista (Briceño-León y

6 Grosfoguel (2008) indica que esta idea estaría sintetizada en la noción de "colonialidad del poder" (Mignolo, 2010), entendida como el proceso de estructuración del sistema-mundo moderno que articula las relaciones económicas, de autoridad, subjetivas y de conocimiento desde la lectura colonial. Para el autor, la colonialidad del poder se trataría de una radicalización de la propuesta poscolonial, que permite transitar hacia lo que ha denominado "decolonialidad". La noción de giro decolonial fue acuñada primeramente por Maldonado-Torres (2005) para dar cuenta del pensamiento que se estructuraría "más allá" del binomio modernidad/ colonialidad, para desmarcarse del pensamiento "mainstream" del colonialismo (Ballestrín, 2013). 
Sonntag, 1999), que pueda desprenderse del modelo cartesiano (y posteriormente newtoniano), base de la conquista de América y de la implantación de la dominación colonial, y que cuestione las pretensiones de objetividad y neutralidad de las ciencias sociales (Lander, 2000) ${ }^{7}$. De esta manera, el poscolonialismo latinoamericano no limita su crítica a las perspectivas dominadoras europeas, sino que genera un cuestionamiento al conjunto de la ciencia y la epistemología contemporáneas, o, como diría Coronil (2000), al globocentrismo como modelo actual de dominación.

Relacionado con lo anterior, una segunda característica genérica del poscolonialismo latinoamericano es el importante papel que se entrega a la experiencia de los sujetos y a la vivencia social en la construcción de la teoría. Esta premisa puede leerse desde por lo menos dos puntos de vista. Por una parte, implica que la teoría debe ser un constructo propio de los investigadores y latinoamericanos, y no una adaptación o imposición externa de investigadores o académicos foráneos ${ }^{8}$, desarrollando así una propuesta que cuestiona lo que Mignolo (1998) ha llamado como "imperialismo" de la teoría social eurocéntrica9. Por otro lado, y más importante, implica una reconstrucción de la relación entre

7 Evidentemente, en este aspecto la teoría poscolonial latinoamericana se entronca y relaciona con perspectivas postestructurales, deconstructivistas y posmodernas, que también han enfatizado en la necesidad de discutir el estatus de la ciencia moderna y su lógica de operación. Siguiendo a Ballestrín (2013), creemos, sin embargo, que la poscolonialidad latinoamericana enfatiza en las consecuencias dominadoras de este proceso, mostrando cómo la ciencia moderna sería una herramienta o instrumento más que permitiría la reproducción de la relación antagónica entre dominador y dominado.

8 Si bien este aspecto no es reconocido explícitamente por los investigadores, si puede ser derivado del hecho de que algunas de las mayores recopilaciones que abordan la perspectiva (Briceño-León y Sonntag, 1998; Lander, 2007; Bialakowsky, 2012) son realizadas exclusivamente por analistas nacidos en América Latina.

9 Según Ballestrín (2013), fue precisamente la incapacidad de romper con la episteme europea (que tenía como referentes principales a Gramsci, Foucault y Derrida) lo que generó un quiebre a principios de los noventa en lo que se conocía como el "Grupo de Estudios Subalternos", en el que coincidían los poscolonialistas latinoamericanos con investigadores poscoloniales de Asia (Ranajit Guha, Partha Chatterjee, Dipesh Chakrabarty, Gayatri Spivak, entre otros) y con otros estudiosos de los estudios culturales, como Stuart Hall o Paul Gilroy. 
el investigador y la investigación, de tal forma que este abandone la mirada en el individuo o la estructura, y construya un abordaje centrado en la comunidad como un espacio común de los sujetos latinoamericanos (Moreno, 1997). Esto permitiría construir una teoría no a partir del pueblo, sino en el pueblo y del pueblo (Alarcón, 2001), generando así una superación de la estructura de distinciones propias del mundo colonial y permitiendo la fusión e identificación entre investigador e investigado como parte de un espacio común. De esta manera, la poscolonialidad se desarrolla desde una alteridad radical, en la que el uno y el otro se reconocen como sujetos en la mirada de lo otro (Grüner, 2012), negándose como totalidad individual (Dussel, 1973, p. 161), profundizando así en la importancia de las relaciones sociales y vivencias en la construcción de la nueva teoría social del continente (Zemelman, 2005; De Sousa Santos, 2009; Moreno, 1997).

Finalmente, una tercera característica de los estudios poscoloniales que los diferenciaría de las perspectivas posmodernas (Alarcón, 2001) es su horizonte normativo orientado a la transformación de la realidad social. Esto implica que la producción teórica se entiende esencialmente como una actividad crítica respecto del pasado, presente y futuro de la sociedad latinoamericana (Sosa, 1998). Este horizonte crítico permitiría la generación de una teoría liberalizadora para toda la comunidad, es decir, tanto para los investigadores como para los investigados (Dussel, 1973). Como indica Lander, esto implica proponer una idea de la liberación de la praxis, o sea, a través de una desnaturalización de las formas canónicas de ser en el mundo (Lander, 2010, p. 28). En algunos casos, este afán crítico implica un reconocimiento explícito del papel de la teoría social en la transformación política de las sociedades latinoamericanas a favor de los oprimidos y desposeídos (Correa, 2012; Sader, 2012), mientras que en otros se entiende como un movimiento de sospecha total, donde lo central es el cuestionamiento constante de las realidades existentes (Alarcón, 2001). Sea como sea, la construcción de una ciencia social crítica de la realidad existente se constituye como un 
elemento central de la perspectiva poscolonial latinoamericana, cuestionando fuertemente el ideal de neutralidad, objetividad y no acción de la teoría social.

\section{Sociedad y teoría social. Los regímenes de producción de conocimiento en las sociedades contemporáneas}

Ahora bien, ¿cuál es la capacidad y alcance explicativo de las teorías poscoloniales respecto de la realidad latinoamericana? ¿Son las teorías poscoloniales latinoamericanas una corriente o paradigma teórico que pueda enraizarse con mayor fuerza que las teorías neoliberales en la realidad del continente? ¿Pueden las teorías poscoloniales llenar el vacío teórico y social generado desde los noventa?

Para responder a estas preguntas debemos retrotraernos a la discusión entre sociedad y teoría social. Una forma de analizar esta relación (evidentemente no la única) es desde la ya clásica división entre estructura y superestructura, que delimita y diferencia el mundo de la economía y las relaciones sociales (estructura) del mundo de las ideas, la ideología y las instituciones que lo sustentan (superestructura). Desarrollada por Marx y Engels (2014 [1845]) en el marco de los análisis del capitalismo industrial y como una forma de destacar la importancia de las relaciones de producción en la realidad social, la distinción entre estructura y superestructura se concibe en el marxismo, desde sus inicios, como una relación ambigua, con rasgos a la vez deterministas e indeterministas (Larraín, 2010). Esta ambigüedad genera una multiplicidad de interpretaciones, perspectivas y acciones, que van a impactar en gran parte de la historia de la sociedad y del pensamiento desde el siglo XX, en el mundo (Hobsbawm, 2011) y en América Latina (Lowy, 2007).

Una de las interpretaciones más reconocidas de la dicotomía entre base y superestructura, desarrollada desde el interior 
del marxismo, es la generada por el pensador italiano Antonio Gramsci. Partiendo de la derrota de los partidos comunistas occidentales durante los años treinta del siglo pasado y por medio de un análisis de la relación entre cultura, sociedad civil y Estado existente en Italia (Santucci, 2005), Gramsci realiza una reconceptualización de la relación entre estructura (economía) y superestructura (ideología) que busca concebir ambos elementos como relacionados histórica y dialécticamente. De esta manera, estructura y superestructura se conciben "como elementos íntimamente vinculados y necesariamente interrelativos y recíprocos" (Gramsci, 1981, p. 186), conformando lo que él llama "bloque histórico" (Gramsci, 1981, p. 202). En este bloque los elementos ideológicos, culturales y económicos se interpenetran y codeterminan, lo que constituye uno de los aportes fundamentales del autor.

Desde nuestra perspectiva, la incorporación de la mirada gramsciana permite ampliar los horizontes analíticos en el estudio de las relaciones entre sociedad y teoría social. Así, la incorporación de la noción de "bloque histórico" y la vinculación dialéctica entre la sociedad (base) y la teoría social (superestructura) permite entender, reconocer y explicitar que los cambios y transformaciones en la teoría social se encuentran relacionados - y en parte determinados - por las condiciones económicas y sociales en que esta teoría se produce y desarrolla. De esta manera, la pregunta por la relevancia o potencialidad de una teoría (que en nuestro caso se circunscribe al análisis del poscolonialismo) se debe relacionar, inevitablemente, con la forma y condiciones en que esta teoría se produce.

Un concepto que cristaliza las formas en las que la teoría se produce es la noción de "régimen de conocimiento" (knowledge regimes). Desarrollada desde finales de los años ochenta a partir de los análisis neoinstitucionalistas, los regímenes de conocimiento pueden definirse como "un conjunto de actores, organizaciones e instituciones que producen y discriminan las ideas que afectan la toma de decisiones y la organización y operación de los regímenes de producción" (Campbell y Pedersen, 2010, p. 169 
[traducción propia]). De esta forma, la idea misma de régimen de conocimiento busca poner de relieve la influencia recíproca que se produciría entre las dinámicas de conocimiento y los modelos de desarrollo político y social de los países, destacando la interdependencia entre teoría social y sociedad en el proceso de construcción de conocimiento.

De esta manera, la introducción de este concepto ilumina dos aspectos novedosos en el análisis de la relación entre sociedad y teoría social. Por una parte, permite entender que los cambios en los paradigmas, modelos o perspectivas teóricas no se producen espontáneamente o a partir de meros procesos revolucionarios o espontáneos, sino que están influidos por la transformación de los modelos de producción de conocimiento de las sociedades (Gibbons, Limonges, Nowotny, Schwartzman, Scott y Trow, 1994) $\mathrm{y}$, por lo tanto, son procesos internos a estas. De esta forma, se otorga importancia al contexto y ambiente en el cual las ideas se producen, entregando así un espacio a los elementos institucionales en la generación y producción de conocimientos, lo que por cierto incluye a la teoría social. Junto con esto, la introducción de la idea de "régimen de conocimiento" permite profundizar en la relación entre los modelos societales y las formas de conocimiento que estos construyen, al menos en dos niveles. En un primer nivel, que podríamos llamar "sistémico", los distintos regímenes de conocimiento se relacionan con distintas formas de organización societal, medios de coordinación entre los actores y formas de diferenciación social (Rammert, 2004) ${ }^{10}$. En un segundo nivel, que po-

10 Rammert (2004) distingue dos regímenes de producción de conocimiento (complementario/especializado y heterogéneo/distribuido) y los asocia a distintas formas de organización societal, diferenciación social y mecanismos de coordinación entre los sujetos. Así, mientras el régimen de producción de conocimiento complementario/especializado se caracterizaría por organizar la sociedad de manera funcional, generar procesos de diferenciación horizontales y mecanismos de coordinación basados en el mercado y la ciencia, los regímenes de conocimiento heterogéneo/distribuido se desarrollarían en sociedades organizadas de manera fragmentaria, con diferenciaciones heterogéneas y donde los mecanismos de coordinación son principalmente las redes y las culturas mixtas. 
dríamos llamar "sociopolítico", los regímenes de conocimiento se asocian a diversos regímenes políticos, a diversas variedades del capitalismo (Campbell y Pedersen, 2010) ${ }^{11}$ y a diferentes modelos de aplicación de políticas públicas (Garcé, 2011) ${ }^{12}$.

La introducción de estos dos elementos - relación entre teorías y modelos de producción de conocimiento, e influencia de regímenes de conocimiento en los modelos de desarrollo y de organización de la sociedad- permite analizar de una manera distinta la relación entre sociedad y teoría social en América Latina, aspecto que desarrollaremos en el siguiente apartado.

\section{Una primera caracterización del régimen de conocimiento en América Latina: universidades, think-tanks y ciencia y tecnología}

Evidentemente, la caracterización del régimen de conocimiento en América Latina requiere de la discusión de elementos organizacionales, institucionales y políticos que interactúan en la producción del conocimiento y la estructuración de las sociedades, lo que requeriría de un amplio debate. En nuestro caso, nos concentraremos en describir, de manera resumida, solo tres elementos que compondrían este régimen: las universidades, los thinks-tanks y las políticas de desarrollo de ciencia y tecnología ${ }^{13}$.

11 Campbell y Pedersen (2010) distinguen cuatro regímenes de conocimiento: i) orientado al mercado (market-oriented knowledge regime); ii) políticamente templado (politically-tempered knowledge regime) iii) orientado al consenso (consensus-oriented knowledge regime) y; iv) tecnocrático estatal (statisttechnocratic knowledge regime). La identificación de estos regímenes se relaciona con las características de las economías (liberal o coordinada) y de los Estados (abiertos o cerrados) que tienen las sociedades.

$12 \mathrm{Al}$ incorporar como variable central del estudio de los regímenes de conocimiento la forma cómo los países construyen las políticas públicas, Garcé (2011) propone pasar de la concepción de régimen de conocimiento (knowledge regime) a la noción de régimen de conocimiento político (political-knowledge regime).

13 Evidentemente, el análisis realiza generalizaciones que no siempre se aplican al conjunto de países de la región. De todas maneras, esto no debiera ser una limitación, 
Respecto de las universidades, es claro que estas constituyen un aspecto central en la configuración y producción de conocimiento, tanto en el mundo en general como en América Latina en particular. Para Brunner y Flisfich (2014), las universidades pueden entenderse como el espacio desde el cual se institucionaliza y racionaliza la cultura, se produce el conocimiento teórico y práctico de las sociedades y se desarrollan los intelectuales como grupo social. Esto implica que la configuración del papel y sentido de las universidades impacta en el régimen de conocimiento que desarrollan las sociedades. Respecto a este punto, el trabajo de Gibbons (1998) inaugura toda una línea de investigación que busca modelar y entender los cambios de las universidades en el nuevo siglo. De manera esquemática, Gibbons argumenta que las universidades estarían cambiando desde un modelo tradicional (que llama Modelo 1), basado en la disciplinariedad, la homogeneidad, la jerarquía y el prestigio académico, a un nuevo modelo (que denomina Modelo 2), en el que el conocimiento de las universidades se organiza en torno a la trans y mutidisciplinariedad, la heterogeneidad en la producción, la reflexibilidad y la colaboración conjunta (Gibbons, 1998; Noworthy, Scott y Gibbons, 2001). Como indican Bleikle y Byrkjeflot (2002), el paso del Modelo 1 al Modelo 2 implica un cambio desde un régimen de conocimiento en el cual el centro de la universidad es la entrega de conocimiento a otro en que lo central es la construcción de conocimiento y la generación de procesos interdisciplinarios de conexión entre la teoría, la práctica y las políticas públicas (Garcé, 2011).

Considerando esto, ¿cómo pueden caracterizarse los regímenes de organización/producción de conocimiento de las universidades de América Latina? De modo sintético, es posible reconocer tres características definitorias de estas organizaciones en la actualidad. En primer lugar, la gran mayoría de las universidades de la región tiene como objetivo central la entrega de conocimien-

pues, en general, existen bastantes coincidencias en las tendencias descritas en el conjunto de estos países. 
to más que la producción de estos. A partir de una caracterización que considera el nivel de publicaciones que realizan las universidades, Brunner y Miranda (2016) indican que el 86\% de las universidades iberoamericanas son puramente docentes o realizan actividades de investigación de manera esporádica, mientras que solo el 1,3\% pueden ser caracterizadas como universidades de investigación (las que, además, son mayoritariamente de España y Portugal). En segundo lugar, análisis comparativos han mostrado que las universidades del continente poseen bajos niveles de competitividad y calidad en el concierto mundial, aun cuando se les compara con organizaciones de otros países con similares niveles de ingreso (Salmi, 2014), lo que repercute en, por ejemplo, menores niveles de desarrollo de patentes y productividad. Finalmente, las universidades latinoamericanas pueden caracterizarse como instituciones sin procesos de gobernanza efectivos, lo que decanta en liderazgos poco relevantes y plataformas de gestión poco eficientes (Bernasconi, 2013), con bajos niveles de conectividad y vinculación en comparación con países de Europa, América del Norte y Asia (Salmi, 2013; Brunner y Villalobos, 2014).

Ahora bien, una segunda institución relevante en la producción de ideas (y por lo tanto, central en la caracterización de los regímenes de conocimiento) en las sociedades contemporáneas son los centros de pensamiento o think-tanks. Más allá de la discusión respecto a su definición y al campo de acción (McGann, 2013; Di Maggio y Powell, 1983) de estas instituciones, lo central en este caso es que, a partir de la reestructuración de las universidades, la globalización del conocimiento y la mediatización de la sociedad, los centros de pensamiento estarían adquiriendo un rol cada vez más relevante en la producción de conocimiento, constituyéndose como agentes comunicantes entre los campos político, económico, de la producción cultural y de los medios de comunicación (Medvetz, 2013); por tanto, un agente central en la configuración de los regímenes de producción de conocimiento. A diferencia de otras formas de cristalización del poder, los 
think-tanks utilizarían un poder difuso - murky power- (Medvetz, 2012) en la configuración del campo del conocimiento, centrado fundamentalmente en la generación de vínculos y relaciones entre los distintos espacios societales y en la difuminación y diseminación de ideas.

En el caso de América Latina, recientes estudios de estas instituciones en el continente (Botto, 2011; Garcé y Uña, 2006) han mostrado que estas organizaciones poseen débiles estructuras organizativas y de financiamiento, que les impiden organizar procesos de investigación largos y que limitan su producción científica principalmente al área de las políticas públicas y la economía aplicada, la que, sin embargo, es poco clara y está basada principalmente en vínculos políticos. Asimismo, un reciente ensayo, que sintetiza una serie de estudios de países de la región que analizan la relación entre universidades y centros de pensamiento (Brunner, Belletini y Arellano, 2014), ha destacado la poca complementariedad que existiría entre ambas organizaciones (en términos de agendas de investigación y recursos humanos) y los limitados campos teóricos y bajos niveles de productividad de los centros de pensamiento del continente. De esta manera, pareciera ser que los centros de pensamiento no estarían adquiriendo un rol central y relacional en la producción del conocimiento en América Latina.

Finalmente, los regímenes de conocimiento también se ven influenciados por las políticas y desarrollos que las naciones realizan en el campo de la ciencia, la tecnología y la producción científica. Como ha destacado Portes (1998), la generación de políticas de desarrollo tecnológico y productivo especializado es un elemento central en la configuración de las relaciones de producción y en las formas de conocimiento que realizan los países. De esta manera, la tecnología, la innovación y el conocimiento se interrelacionan en la producción de un determinado régimen de conocimiento (Rammert, 2004), que a su vez impacta en las relaciones sociales, políticas y de producción de las sociedades contemporáneas (Campbell y Pedersen, 2010). En este sentido, se 
ha postulado que la generación de procesos de desarrollo científico y tecnológico con alto nivel de adaptación, flujos constantes y circuitos virtuosos entre conocimiento y práctica serían las claves del desarrollo científico contemporáneo (Nonaka y Takeuchi, 1995), lo que permitiría la estructuración y crecimiento de la producción de ideas.

Información secundaria disponible muestra que, en el caso de las sociedades latinoamericanas, el nivel de desarrollo de la ciencia, tecnología y producción es en general bastante precario e incipiente. Tanto la participación de las industrias de ingeniería en el Producto Interno Bruto de los países de América Latina como la inversión en I+D como porcentaje del PIB $(0,4)$ son sustancialmente más bajos que los países emergentes de Asia $(1,21)$ y que otros países desarrollados basados en recursos naturales $(1,89)$ (Ocampo, 2011, p. 26). Asimismo, el modelo de desarrollo aplicado en los países del continente no ha potenciado la construcción de mecanismos de diversificación productiva, que permitan desarrollar niveles complejos de innovación y desarrollo científico (CEPAL, 2007), sino que han fomentado una cierta reprimarización del modelo de desarrollo (Cortés, 2012), el que se ha desarrollado de manera aislada a los espacios prácticos, con muy poca vinculación de las organizaciones de trabajadores y de manera bastante vertical (Mourao, 2014), sin potenciar en mayor medida la construcción de vínculos o procesos horizontales y fluidos entre conocimiento y producción. Además, la producción de conocimiento, especialmente en el ámbito de las ciencias sociales, no se ha desarrollado mayormente en el continente. Como muestra Beigel (2013), el proceso de internacionalización, mercantilización y estandarización del conocimiento mediante índices cientométricos ha generado un abandono y despotenciamiento de las revistas del continente, ya que los investigadores tienden a preferir discutir y publicar en espacios (especialmente norteamericanos y europeos) que le entregan mayor estatus, prestigio e incentivos, lo que ha disminuido tanto la cantidad de citas realizadas entre papers desarrollados en el continente (de 
11,7\%, en el periodo 1993-1995, a 6,9\%, en el periodo 2003-2005), así como el nivel de aporte en la producción de las ciencias sociales de revistas del continente, y que se ubican en el nivel de África y bajo Asia y Oceanía (y por supuesto, también Europa y Norteamérica). De esta manera, los incentivos y características con las que se ha implementado el capitalismo académico (Slaughter y Leslie, 1997) en el continente también han impactado en la capacidad de producción de teoría social latinoamericana.

De esta forma, y a modo de síntesis, podemos constatar que en los tres aspectos revisados (universidades, think-tanks y ciencia y tecnología) el régimen de conocimiento de América Latina es bastante débil y precario en términos de la creación, producción y potenciamiento de conocimiento. De esta manera, es claro que las instituciones y campos para la construcción teórica en el continente son acotados, estructurados y condicionados por distintos factores económicos y culturales, lo que evidentemente condiciona y en parte determina las características y potencialidades del desarrollo del conocimiento en general, y de las teorías sociales en particular, en Latinoamérica.

\section{Reflexiones finales. Poscolonialidad, teoría social y sociedad en América Latina a la luz del régimen de conocimiento del continente}

La caracterización de los principales elementos del régimen de conocimiento de los países latinoamericanos permite replantear la discusión entre sociedad y teoría social, así como la respuesta poscolonial al vacío teórico del continente, aportando de esta manera a la pregunta por la emergencia, relevancia y capacidad interpretativa de la teoría social contemporánea en el continente.

El análisis ha mostrado que las debilidades del continente en términos de su producción de conocimiento están relacionadas con un régimen de conocimiento precario, débil y no desarro- 
llado. Desde este punto de vista, el problema de la producción de conocimiento y de la teoría social en el continente no tiene su origen fundamental en su contenido (la lógica racional, europeizante y cientificista con que se produce la teoría social, como afirmaría parte de la teoría poscolonial), sino en la debilidad del modelo de desarrollo, forma de producción de ideas y el régimen de conocimiento latinoamericano a partir del cual se estructura (o configura) la teoría social, que no deja espacio, incentiva ni permite la emergencia y construcción de teorizaciones potentes ${ }^{14}$. El problema del "vacío teórico" sería entonces un problema primariamente (tanto en su sentido contenido como temporalmente) estructural, más que un problema conceptual (como indican las teorías poscoloniales).

Por ello, este análisis desafía a la teoría poscolonial. Tal como reconoce Lander (2000), la mirada poscolonial parte de la premisa de que es necesario abandonar la economía para la construcción de una teoría social latinoamericana, ya que esta (la economía) asume en sí misma la visión liberal de la sociedad ${ }^{15}$. Nuestro análisis revela, sin embargo, todo lo contrario: si queremos entender el vacío teórico del continente y su falta de construcción propia durante las últimas décadas, debemos estudiar en profundidad los regímenes de conocimiento débiles, poco eficientes y precarizados del continente, que no otorgan mayor importancia a los procesos de innovación analítica y desarrollo teórico en los distintos países de América Latina. Tal como dice Marcia Rivera, "resulta muy

14 Entenderemos como teoría social potente a aquella que podría tener una potencialidad explicativa de alto impacto, esto es, que puede ser un marco de referencia teórico válido y utilizado para la comprensión de determinadas realidades sociales. Desde este punto de vista, la potencia se asocia más a la utilidad y utilización de la teoría que a su correspondencia con la realidad.

15 Es importante mencionar que la perspectiva de Lander no necesariamente es compartida por todo el universo de intelectuales poscoloniales. A pesar de esto, y como hemos intentado mostrar, la perspectiva poscolonial parte del diagnóstico de la necesidad de repensar teórica y epistémicamente el entendimiento de las sociedades latinoamericanas y las herramientas analíticas con las que estudiamos esta realidad. Se trata, de esta manera, de un punto de partida que tiene en su base la crítica a la imposición epistémica, teórica y metodológica eurocéntrica/cientificista. 
difícil separar los problemas sustantivos de la investigación -incluyendo los temas de construcción teórica, transdisciplinariedad, diálogo con las ciencias duras, relevancia política- de los marcos institucionales en los cuales se produce" (Rivera, 1999, p. 131) ${ }^{16}$, lo que implica reconocer la importancia de trabajar teóricamente el problema de la teoría social latinoamericana a partir de la relación dialéctica entre sociedad (estructura) y teoría (superestructura).

¿Cuál es entonces la alternativa? Sin pretender exhaustividad, creemos que es posible repensar una teoría social potente desde investigadores latinoamericanos que, reconociendo las particularidades del continente, su formación y desarrollo científico, tengan como horizonte analítico el entendimiento de la realidad social global. Esto implica, a contrapelo de las miradas poscoloniales (aunque en este punto también comparten con las llamadas teorías posmodernas), reconocer y validar la existencia de una ciencia universal (Sokal y Bricmont, 1999), entendida eso sí como una práctica social que "nunca es enteramente cierta y muy a menudo es bastante falsa, pero tiene por lo general más posibilidades de ser cierta que las teorías no científicas y, por lo tanto, es razonable aceptarla hipotéticamente" (Russell, 1992, p. 113). Asimismo, parece relevante repensar la relación entre teoría y realidad, buscando teorizaciones que puedan resultar razonables (y, podríamos agregar, crecientemente razonables) para explicar la realidad actual de los países del continente (Sokal, 2007) ${ }^{17}$.

16 Desde nuestro punto de vista, el análisis de Rivera (1999) contiene una contradicción fundamental. A pesar de que reconoce, al igual que nosotros, la centralidad de los aspectos institucionales, económicos y estructurales para entender el vacío teórico de las ciencias sociales, señala, a renglón seguido, que la única solución para repensar el oficio sociológico y de los cientistas sociales es realizar un proceso de construcción de conocimiento endógeno, que no sea automáticamente copiado de Europa o Estados Unidos. De esta manera, impone una solución única (el poscolonialismo) a un problema con múltiples salidas (la debilidad de la institucionalidad de las ciencias sociales).

17 Esto no implica adoptar necesariamente una posición popperiana o verificacionista respecto de la realidad. Se trata más bien de seguir un realismo modesto (Kitcher, 2001; Sokal y Bricmont, 2001) que, basado en un racionalismo moderado, entienda el rol de las teorías sociales como una interpretación plausible 
De forma implícita, la adopción de estas premisas epistémico-teóricas implica abandonar la idea misma de "teoría social latinoamericana" y reemplazarla por la de "teoría social realizada por latinoamericanas y latinoamericanos". Esta teoría propuesta por latinoamericanas y latinoamericanos (en un sentido no esencialista ${ }^{18}$ ) podría ser una herramienta útil para analizar las sociedades latinoamericanas, pero también para estudiar la realidad social "en abstracto", compitiendo y luchando de igual modo con los análisis sociales realizados por europeos, asiáticos y norteamericanos ${ }^{19}$. De esta manera, más que negar la existencia de la ciencia contemporánea - y como afirma acertadamente Fernanda Beigel (2013), de generar una desconexión con el pensamiento occidental - como lo señalan los poscolonialistas, creemos que la construcción de una teoría social generada desde América Latina debe fundarse en el reconocimiento al ingreso en el campo científico mundial (con sus códigos, epistemes y métricas establecidas), entendiendo que en este proceso es posible desarrollar un proceso de lucha entre teorías sociales, en el que las y los latinoamericanos pueden disputar estos espacios, aunque reconociendo su posición desventajada por las condiciones de la estructura social.

de la realidad y que hace sentido a las personas que vivencian esta realidad. Para una descripción más detallada del realismo modesto, ver Villalobos (2014).

18 Aunque no hay espacio para profundizar en este aspecto, las interrogantes aquí planteadas nos llevan a preguntarnos sobre el estatus mismo que lleva a cualquier analista a ser considerado como investigadora o investigador "latinoamericano", cuestionando la centralidad de la nacionalidad o el lugar de estudios como claves o llaves para la imposición de este atributo. Como ha mostrado Ballestrín (2013) para el caso de los teóricos del giro decolonial, en la mayoría de los casos se trata de investigadores de nacionalidades latinoamericanas, aunque su ejercicio profesional se desarrolla, en no pocos casos, en universidades norteamericanas. Repensar las etiquetas que permiten clasificar a los teóricos es, pues, otro de los desafíos de la propia teoría social.

19 Así, el punto a resaltar es el siguiente: si las teorías sociales de pensadores contemporáneos (europeos), como Giddens, Foucault, Bourdieu, Beck o tantos más, han sido la base para el estudio de sociedades latinoamericanas, ¿por qué las teorías sociales de pensadores nacidos en América Latina no podrían servir para el análisis de sociedades europeas y no exclusivamente para entender y teorizar sobre las sociedades latinoamericanas? 
Para desarrollar este proceso es claro que se necesita reforzar, consolidar y profundizar los regímenes de conocimiento actuales del continente. Así, potenciar la investigación en las universidades, mejorar la relación entre centros de pensamiento, universidades y sociedad civil, mejorar la calidad de la investigación en el continente, priorizar instancias de acumulación intelectual, promover y potenciar discusiones sobre la realidad social latinoamericana y mejorar las condiciones de trabajo de los investigadores son, entre otros, desafíos de corto y mediano plazo para realizar esta tarea. Esto nos permitirá construir una teoría social desde América Latina, "racionalista pero no dogmática, con mentalidad científica pero no cientificista, amplia de miras pero no frívola" (Sokal y Bricmont, 1999, p. 229), que permita, al mismo tiempo, teorizar respecto del continente y respecto del conjunto de las transformaciones globales.

\section{Referencias}

Alarcón, L. (2001). Perspectivas de la sociología latinoamericana. Sociología de la alteridad en el siglo XXI. Cinta de Moebio, 13(1), 85-103.

Ballestrín, L. (2013). América Latina e o giro decolonial. Revista Brasileira de Ciencia Política, 11, 89-117.

Beigel, F. (2013). Centros y periferias en la circulación internacional de conocimiento. Revista Nueva Sociedad, 245(5), 110-123.

Bernasconi, A. (2008). La crisis del modelo latinoamericano de universidad, en JJ. Brunner y C. Peña (eds.), Reforma de la educación superior (pp. 47-84). Santiago: Ediciones Universidad Diego Portales.

Bernasconi, A. (2013). Rankings expose weakness in research and governance. University World News, Issue 275.

Bleiklie, I. \& Byrkjeflot, H. (2002). Changing Knowledge Regimes: Universities in a New Research Environment. Higher Education, 44(3-4), 519-532.

Bortoluci, J. \& Jansen, R. (2013). Toward a postcolonial sociology: The view from Latin America. Political Power and Social Theory, 24, 199-229. 
Briceño-León, R. y Sonntag, H. (1998). La sociología en América Latina entre pueblo, época y desarrollo, en R. Briceño-León y H. Sonntag, H. (eds.), Pueblo, época y desarrollo: La sociología en América Latina (pp. 11-26). Caracas: Ediciones Nueva Sociedad.

Briceño-León, R. y Sonntag, H. (1999). El legado de la sociología, la promesa de las ciencias sociales. Caracas: Ediciones Nueva Sociedad.

Brunner, J. J. y Flisfich, A. (2014). Los intelectuales y la institucionalización de la cultura. Santiago: Ediciones Universidad Diego Portales.

Brunner, J. J. y Miranda, D. (2016). Educación superior en Iberoamérica. Reporte 2016. Santiago: CINDA.

Brunner, J. J. y Villalobos, C. (2014). Políticas de Educación Superior en Iberoamérica 2009-2013. Santiago: Universidad Diego Portales.

Brunner, J. J., Belletini, O. y Arellano, A. (2014). Más saber América Latina. Potenciando el vinculo entre think-tanks y universidades. Santiago: Think-Tank Iniciative.

Botto, M. (2011). Think-tanks en América Latina: Radiografía comparada de un nuevo actor político, en N. Correa y E. Mendizábal (eds.), Vínculos entre conocimiento y política: el rol de la investigación en el debate público de América Latina (pp. 83-112). Lima: Universidad del Pacífico.

Campbell, J. \& Pedersen, O. (2010). Knowledge regimes and comparative political economy, en D. Beland y R. Cox (eds.), Ideas and politics in social science research (pp. 167-190). Oxford: Oxford University Press.

CEPAL (2007). La hora de la igualdad. Brechas por cerrar, caminos por abrir. Santiago: CEPAL.

Cortés, A. (2012). Modernización, dependencia y marginalidad: itinerario conceptual de la sociología latinoamericana. Sociologías, 14(29), 214-238.

Cortés, A. (2012). La reprimarización del modelo de desarrollo chileno. Oikos, 11(1), 66-89.

Correa, R. (2012). Speech in commemoration of FLACSO's 50 ${ }^{\text {th }}$ Anniversary, en A. Bialakowsky (comp.), Latin American Critical Thought: Theory and Practice (pp. 209-218). Buenos Aires: CLACSO.

Costa, S. (2006). Dois Atlanticos: Teoria social, anti-racismo e cosmopolitismo. Belo Horizonte: Editora UFGM. 
De Sierra, G., Garretón, M. A., Murmis, M. y Trindade, H. (2007). Las ciencias sociales en América Latina, en H. Trindade (coord.), Las Ciencias Sociales en América Latina. Una perspectiva comparada (pp. 17-54). México D.F.: Siglo XXI Editores.

De Sousa Santos, B. (2009). Epistemología del sur. México D.F.: Siglo XXI Editores.

De Sousa Santos, B. (2010). Descolonizar el saber, reinventar el poder. Montevideo: Ediciones Trilce.

Di Maggio, P. \& Powell, W. (1983). The iron cage revisited: Institutional isomorphism and collective rationality in organization fields. American Sociological Review, 48(2), 147-160.

Dos Santos, T. (1998). La teoría de la dependencia: un balance histórico y teórico, en F. López (ed.), Los retos de la globalización. Ensayos en homenaje a Theotonio Dos Santos. Caracas: UNESCO.

Dussel, E. (2000). Europa, modernidad y eurocentrismo, en E. Lander (ed.), La colonialidad del saber. Eurocentrismo y Ciencias Sociales. Perspectivas latinoamericanas (pp. 41-54). Buenos Aires: CLACSO.

Dussel, E. (1973). Para una ética de la liberación latinoamericana. Buenos Aires: Siglo XXI Ediciones.

Ffrench-Davis, R. (2014). Chile entre el neoliberalismo y el crecimiento con equidad. Cuarenta años de políticas económicas y sus lecciones para el futuro. Santiago: JC Sáez Editores.

Garcé, A. (2011). Political-Knowledge regimes. Developing a new concept from the study of three changes in public policy during the government of Tabaré Vasquez in Uruguay (20052011). Politics \& Ideas, 8, 1-18

Garcé, A. y Uña, G. (2006). Think-tanks y políticas públicas en Latinoamérica. Dinámicas globales y realidades regionales. Buenos Aires: Prometeo.

Garretón, M. A. (2014). Las Ciencias Sociales en la trama de América Latina y Chile (manuscrito).

Garretón, M. A. Cavarozzi, M., Cleaves, P., Gereffi, G. y Hartlyn, J. (2004). América Latina en el Siglo XXI. Hacia una nueva matriz sociopolítica. Santiago: LOM.

Gibbons, M. (1998). Higher Education Relevance in the $21^{\text {st }}$ Century. Documento presentado en la Conferencia Mundial de Educación Superior, París, 5-9 de octubre. 
Gibbons, M., Limoges, C., Nowotny, H., Schwartzman, S., Scott, P. \& Trow, M. (1994). The new production of knowledge. The dynamics of science and research in contemporary society. Londres: Thousand Oaks.

González, M. (2014). New modes of governance of Latin American higher education: Chile, Argentina and Mexico. Bordón, 66(1), 137-150.

Gramsci, A. (1981). Cuadernos de la cárcel. Tomo IV, México D.F.: Nueva Era.

Grosfoguel, R. (2008). Para descolonizar os estudos de economía política e os estudos pós-coloniais: transmodernidades, pensamento de fronteira e colonialidade global. Revista Crítica de Ciencias Sociais, 80(2), 115-147.

Grüner, E. (2012). The gaze of/on the other. Sartre, Pasolini and the European cultural fascination for the Third World, en A. Bialakowsky (comp.), Latin American Critical Thought: Theory and Practice (pp. 105-117). Buenos Aires: CLACSO.

Hobsbawm, E. (2011). Como cambiar el mundo. Marx y el marxismo. 18402011. Buenos Aires: Crítica.

Kitcher, P. (2001). Science, truth and democracy. New York: Oxford University Press.

Lander, E. (2000). Ciencias Sociales. Saberes coloniales y eurocéntricos, en E. Lander (ed.), La colonialidad del saber. Eurocentrismo y Ciencias Sociales. Perspectivas latinoamericanas (pp. 11-40). Buenos Aires: CLACSO.

Lanz, R. (1998). La sociología que viene. Pensar después de la posmodernidad, en R. Briceño-León y H. Sonntag (eds.), Pueblo, época y desarrollo: La sociología en América Latina (pp. 79-85). Caracas: Nueva Sociedad.

Larraín, J. (2007). El concepto de ideología. Vol. I. Carlos Marx. Santiago: LOM.

López-Segrera, F. (2000). Abrir, impensar y redimensionar las Ciencias Sociales en América Latina y el Caribe. ¿Es posible una ciencia social no eurocéntrica en nuestra región?, en E. Lander (ed.), La colonialidad del saber. Eurocentrismo y Ciencias Sociales. Perspectivas latinoamericanas (pp. 177-200). Buenos Aires: CLACSO.

Lowy, M. (2007). El marxismo en América Latina. Antología, desde 1909 hasta nuestros días. Santiago: LOM. 
Maldonado-Torres, N. (2007). Sobre la colonialidad del ser: contribuciones al desarrollo de un concepto, en S. Castro-Gómez y R. Grosfoguel (coords.), El giro decolonial: reflexiones para una diversidad epistémica más allá del capitalismo global. Bogotá: Siglo del Hombre Editores, Universidad Central, Instituto de Estudios Sociales Contemporáneos, Pontificia Universidad Javierana, Instituto Pensar.

Marx, K. y Engels, F. (2014 [1845]). La ideología alemana. Madrid: Akal.

McGann, J. (2013). Global go think tanks report and policy advise. Think tanks and civil societies program. Pensylvannia: University of Pensylvannia.

Medvetz, T. (2013). Murky power: Think-tanks as boundary organizations, en D. Courpasson, D. Golsorkhi \& J. Sallaz (eds.), Re-thinking power in organizations, institutions and markets. Londres: Emerald Group Publisher.

Medvetz, T. (2012). Think tanks in America. Chicago: University of Chicago Press.

Mignolo, W. (1998). Postoccidentalismo: el argumento desde América Latina, en S. Castro-Gómez y E. Mendieta (coords.), Teoría sin disciplina: latinoamericanismo, poscolonialidad y globalización en el debate. México: Miguel Ángel Porrúa.

Mignolo, W. (2000). La colonialidad a lo largo y ancho. El hemisferio occidental en el horizonte colonial de la modernidad, en E. Lander (ed.), La colonialidad del saber. Eurocentrismo y Ciencias Sociales. Perspectivas latinoamericanas (pp. 55-86). Buenos Aires: CLACSO.

Mignolo, W. (2010). Desobediencia epistémica: retórica de la modernidad, lógica de la colonialidad y gramática de la descolonialidad. Argentina: Ediciones del Signo.

Moreno, A. (1997). Desencuentro de mundos. Revista Heterotropía, 6, 1137.

Mourao, V. (2014). La importancia de la participación de los trabajadores en la conformación de la política de ciencia, tecnología e innovación. Cuadernos de Educación, 29(1), 18-29.

Nonaka, I. \& Takeuchi, H. (1995). The Knowledge-creating Company. How Japanese companies create the dynamics of innovations. New York: Oxford University Press.

Noworthy, H., Scott, P. \& Gibbons, M. (2001). Re-thinking science. Knowledge and the Public in an Age of University. Cambridge: Polity. 
Ocampo, J. (2011). Macroeconomía para el desarrollo: políticas anticíclicas y transformación productiva. CEPAL Review, 104(1), 7-35.

Packenhaum, R. (1992). The Dependency Movement: Scholarship and Politics in Development Studies. Cambridge: Cambridge University Press.

Portes, A. (2004). La sociología en el continente: convergencias pretéritas y una nueva agenda de alcance medio. Revista Mexicana de Sociología, 66(3), 1-37.

Portes, A. (1998). El neoliberalismo y la Sociología del Desarrollo: Tendencias emergentes y efectos inesperados. Perfiles Latinoamericanos, 13, 9-53.

Quijano, A. (2012). Live Well. Between the development and the descolonianity of power, en A. Bialakowsky (comp.), Latin American Critical Thought: Theory and Practice (pp. 15-26). Buenos Aires: CLACSO.

Rammert, W. (2004). Two styles of knowing and knowledge regimes: Between explicitation and exploration under conditions of functional specialization or fragmental distribution, Berlin: Technical University Technology Studies Working Papers.

Riesco, M. (2012). Parto de un siglo. Una mirada al mundo desde la izquierda de América Latina. Santiago: Ediciones USACH.

Rivera, M. (1999). Reinventando el oficio: el desafío de reconstruir la investigación en ciencias sociales en América Latina hacia el próximo milenio, en R. Briceño-León y H. Sonntag (eds.), Pueblo, época y desarrollo: La sociología en América Latina (pp. 119-133). Caracas: Nueva Sociedad.

Russell, B. (1992). La evolución dentro de mi pensamiento filosófico. Madrid: Alianza.

Sader, E. (2012). Orphans of strategy?, en A. Bialakowsky (comp.), Latin American Critical Thought: Theory and Practice (pp. 219-236). Buenos Aires: CLACSO.

Salmi, J. (2014). Las reformas de educación superior en Iberoamérica en una perspectiva global. Documento de Trabajo, Centro de Políticas Comparadas en Educación, Universidad Diego Portales.

Salmi, J. (2013). Formas exitosas de gobierno universitario en el mundo. CyD Foundation Study. 
Santucci, A. (2005). Gramsci, Santiago: LOM.

Slaugther, S. \& Leslie, L. (1997). Academic Capitalism: Politics, Policies and the Entrepreneurial University. Baltimore: Johns Hopkins.

Sokal, A. (2007). Más allá de las imposturas intelectuales. Barcelona: Paidós.

Sokal, A. \& Bricmont, J. (2001). Defense of a modest realism. Conferencia dictada por Alan Sokal en la Universidad de Bielefeld el 18 de junio de 2001.

Sokal, A. y Bricmont, J. (1999). Imposturas intelectuales. Barcelona: Paidós.

Sosa, R. (1998). Los retos de la sociología latinoamericana: algo de lo que tenemos, algo de lo que buscamos, en R. Briceño-León y H. Sonntag (eds.), Pueblo, época y desarrollo: La sociología en América Latina (pp. 51-55). Caracas: Nueva Sociedad.

Trindade, H. (2007). Las Ciencias Sociales en América Latina. Una perspectiva comparada, México D.F.: Siglo XXI Editores.

Villalobos, C. (2014). Los conflictos sociales en la sociedad contemporánea. Apuntes conceptuales y metodológicos desde el realismo modesto. Cuadernos de Trabajo Social, 12(1), 79-93.

Zemelman, H. (2005). Voluntad de conocer. El sujeto y su pensamiento en el paradigma crítico. México D.F.: Anthropos. 